\title{
On the computation of space-time correlations by large-eddy simulation
}

\author{
Guo-Wei He \\ Center for Turbulence Research, NASA Ames Research Center/Stanford University, MS 19-44, Moffett Field, \\ California 94035 \\ and LNM, Institute of Mechanics, Chinese Academy of Sciences, Beijing 100080, China \\ Meng Wang ${ }^{\mathrm{a})}$ \\ Center for Turbulence Research, NASA Ames Research Center/Stanford University, MS 19-44, Moffett Field, \\ California 94035 \\ Sanjiva K. Lele \\ Departments of Aeronautics and Astronautics and Mechanical Engineering, Durand Building, \\ Stanford University, Stanford, California 94305-4035
}

(Received 31 December 2003; accepted 18 May 2004; published online 15 September 2004)

The effect of subgrid-scale (SGS) modeling on velocity (space-) time correlations is investigated in decaying isotropic turbulence. The performance of several SGS models is evaluated, which shows superiority of the dynamic Smagorinsky model used in conjunction with the multiscale large-eddy simulation (LES) procedure. Compared to the results of direct numerical simulation, LES is shown to underpredict the (un-normalized) correlation magnitude and slightly overpredict the decorrelation time scales. This can lead to inaccurate solutions in applications such as aeroacoustics. The underprediction of correlation functions is particularly severe for higher wavenumber modes which are swept by the most energetic modes. The classic sweeping hypothesis for stationary turbulence is generalized for decaying turbulence and used to analyze the observed discrepancies. Based on this analysis, the time correlations are determined by the wavenumber energy spectra and the sweeping velocity, which is the square root of the total energy. Hence, an accurate prediction of the instantaneous energy spectra is most critical to the accurate computation of time correlations.

(C) 2004 American Institute of Physics. [DOI: 10.1063/1.1779251]

\section{INTRODUCTION}

Space-time correlations or their Fourier transformations, wavenumber-frequency spectra are the simplest space-time statistics of turbulent flows. They are of interest in fundamental turbulence research as well as in various practical applications. For example, according to Lighthill's theory, ${ }^{1,2}$ the acoustic intensity radiated by a turbulent flow depends on the two-time, two-point velocity correlations. In wallbounded flows, the calculation of flow-induced vibration and sound requires the wavenumber-frequency spectra of wallpressure fluctuations as a forcing function input to structural models. ${ }^{3}$ In boundary-layer receptivity problems the wavenumber-frequency spectra of free-stream disturbances are critical to the transition from laminar to turbulent flows. ${ }^{4}$ In turbulence control and drag reduction applications, ${ }^{5}$ the space-time characteristics of turbulent fluctuations have been used as control inputs for the blowing and suction by actuators. Further applications can be found in, for example, particle dispersion ${ }^{6}$ and predictability. ${ }^{7}$

In recent years there has been an increasing interest in applying large-eddy simulation (LES) to solve flow problems, such as those mentioned above, in which the spacetime characteristics are important. The existing subgrid scale (SGS) models are, however, mostly constructed to predict spatial statistics such as energy spectra. $^{8}$ It is not clear

\footnotetext{
a)Telephone: (650) 604-4727; fax: (650) 604-0841; electronic mail: wangm@stanford.edu
}

whether these models can lead to accurate predictions of the space-time correlations, or frequency contents at individual wavenumbers. Hence, the accurate prediction of space-time correlations presents a new challenge for SGS modeling. This is particularly important to aeroacoustic predictions because, for a given frequency, only the spectral element of the source field corresponding to the acoustic wavenumber in a given direction can radiate sound in that direction. ${ }^{9}$ The radiation represents a very small fraction of flow energy, and is extremely susceptible to numerical and modeling errors.

For brevity, we henceforth refer to the two-time, twopoint correlation of the velocity field simply as time correlation. It can be equivalently expressed by a two-time correlation of velocity Fourier modes in spectral space

$$
C(k, \tau)=\left\langle u_{i}(\mathbf{k}, t) u_{i}(-\mathbf{k}, t+\tau)\right\rangle,
$$

or its normalized form

$$
R(k, \tau)=\frac{\left\langle u_{i}(\mathbf{k}, t) u_{i}(-\mathbf{k}, t+\tau)\right\rangle}{\left\langle u_{i}(\mathbf{k}, t) u_{i}(-\mathbf{k}, t)\right\rangle} .
$$

A previous study by He, Rubinstein, and Wang, ${ }^{10}$ compared the normalized time correlations, or correlation coefficients, in forced isotropic turbulence calculated by direct numerical simulation (DNS) and LES using the spectral eddy-viscosity model of Chollet and Lesieur. ${ }^{11}$ The comparison shows that the LES overpredicts decorrelation time scales.

In the present work, we examine the SGS modeling effects on time correlations further and from a different per- 
spective. The objectives are twofold. The first objective is to evaluate the performance of several popular SGS models in terms of time correlations by comparison with DNS solutions. The models considered are the spectral eddy-viscosity model ${ }^{11}$ the classic Smagorinsky model, ${ }^{12}$ the dynamic Smagorinsky model, ${ }^{13}$ and the multiscale LES method of Hughes, Mazzei, and Oberai, ${ }^{14}$ in conjunction with the dynamic Smagorinsky model. A second objective is to analyze the observed discrepancies based on the sweeping hypothesis, ${ }^{15}$ in order to identify the sources of timecorrelation errors and their influence on aeroacoustic calculations. Unlike the previous study, ${ }^{10}$ the evaluations and analysis are carried out for the un-normalized time correlations, not the normalized ones, since the former are the ones actually used in the computation of sound power spectra. Furthermore, we consider decaying homogeneous isotropic turbulence so that the results are not affected by forcing. In contrast to the stationary turbulence considered earlier, the time correlations are dependent on both time separations and starting time. Two different starting times will be chosen, one during the initial period characterized by the decay of energy-containing eddies via energy propagation to small scales, and another during the final decay period dominated by viscous effects.

The analysis starts with a generalization of Kraichnan's sweeping hypothesis ${ }^{15}$ from stationary turbulence to decaying turbulence. This involves replacing a constant convection velocity by a time-dependent one in a simple kinematic model. The solution of the kinematic model defines a timedependent sweeping velocity. Kraichnan's sweeping hypothesis is the foundation of the turbulence theory on time correlations. Kaneda and Gotoh ${ }^{16}$ and Kaneda ${ }^{17}$ developed the Lagrangian renormalization group theory and the Taylor expansion technique for time correlations. Rubinstein and Zhou ${ }^{18}$ used the sweeping hypothesis to formulate the scaling law of sound power spectra.

Finally, the present analysis on time correlations will be used to shed some light on the ability of LES to predict sound power spectra. This is an important issue given the increasing use of LES for aeroacoustic prediction in recent years (e.g., Ref. 19). A previous study of SGS modeling effects by Piomelli, Streett, and Sarkar, ${ }^{20}$ is focused on the spatial statistics of Lighthill source terms. Other evaluations, ${ }^{21-23}$ made directly on acoustic fields, unavoidably have to cope with the numerical errors caused by the truncation of the source region. ${ }^{24,25}$ Instead, we will discuss the influences of SGS modeling on the accuracy of sound prediction through an analysis of time correlations in the Lighthill framework coupled with the quasinormal closure assumption.

\section{NUMERICAL RESULTS}

A decaying homogeneous isotropic turbulence in a cubic box of side $2 \pi$ is simulated by DNS with grid size $256^{3}$ and LES with grid size $64^{3}$. A standard pseudospectral method is used, in which spatial differentiation is made by the Fourier spectral method, time advancement is made by a secondorder Adams-Bashforth method with the same time steps for both DNS and LES, and molecular viscous effects are accounted for by an exponential integrating factor. All nonlinear terms are dealiased with the two-thirds rule.

The following SGS models are used in the LES.

(1) The spectral eddy-viscosity model: We use the Chollet-Lesieur standard form for the spectral eddy viscosity, ${ }^{11}$ where the cutoff energy is evaluated from the LES.

(2) The Smagorinsky model: ${ }^{12}$ The Smagorinsky constant is $C_{s}=0.22$ and the filter width is set equal to the inverse of the largest effective wavenumber $k_{c}=21$.

(3) The dynamic Smagorinsky model: ${ }^{13}$ The Smagorinsky coefficients are determined by the Germano identity. The grid filter width is $k_{c}^{-1}$ and the test filter width is taken as $2 k_{c}^{-1}$.

(4) The multiscale LES method ${ }^{14}$ with dynamic SGS model: We decompose the filtered Navier-Stokes equations into large-scale equations for the lower one-half Fourier modes and small-scale equations for the remaining half Fourier modes. The dynamic Smagorinsky model is applied to the small scale equations.

The initial condition for DNS is an isotropic Gaussian field with energy spectrum

$$
E(k, 0) \propto\left(k / k_{0}\right)^{4} \exp \left[-2\left(k / k_{0}\right)^{2}\right],
$$

where $k_{0}=4.68$ is the wavenumber corresponding to the peak of the energy spectrum. The shape of the energy spectrum excludes the effects of the box size. The initial Reynolds number based on Taylor's microscale is 127.4. The initial condition for LES is obtained by filtering the initial DNS velocity fields with filtering wavenumber $k_{c}=64 / 3 \approx 21$. Therefore, the initial LES and filtered DNS velocity fields are exactly the same. At early stages, the LES and DNS velocity fields are highly correlated due to the same initial conditions. Therefore, the time correlations of the LES velocity field are nearly the same as those of the DNS field. As time progresses, the LES fields become decorrelated from the DNS fields. The difference in time correlations between the LES and DNS velocity fields are then observed. Therefore, we first advanced the DNS and LES velocities in time to decorrelate them before starting to calculate the time correlations.

The energy spectra at $t=0.5$ and $t=4.0$ are presented in Fig. 1. Generally speaking, the LES spectra are in good agreement with the DNS result at low wavenumbers but drop off faster at higher wavenumbers. The decay of the total resolved energy with time is presented in Fig. 2. The results from LES with all SGS models follow the DNS results with some deviations throughout the entire time range. They exhibit excessive dissipation before the time $t=1.5$ (the energy propagation range) and insufficient dissipations after $t=1.5$ (the final decay range). In both Figs. 1 and 2, the classic Smagorinsky model results are clearly the least accurate. The performances of the other three SGS models are comparable judged for the entire time range shown in Fig. 2. For $t$ $\leqslant 1.5$, however, the multiscale LES with dynamic model is superior compared with the dynamic Smagorinsky model and spectral eddy-viscosity model. The latter two yield similar solutions. 

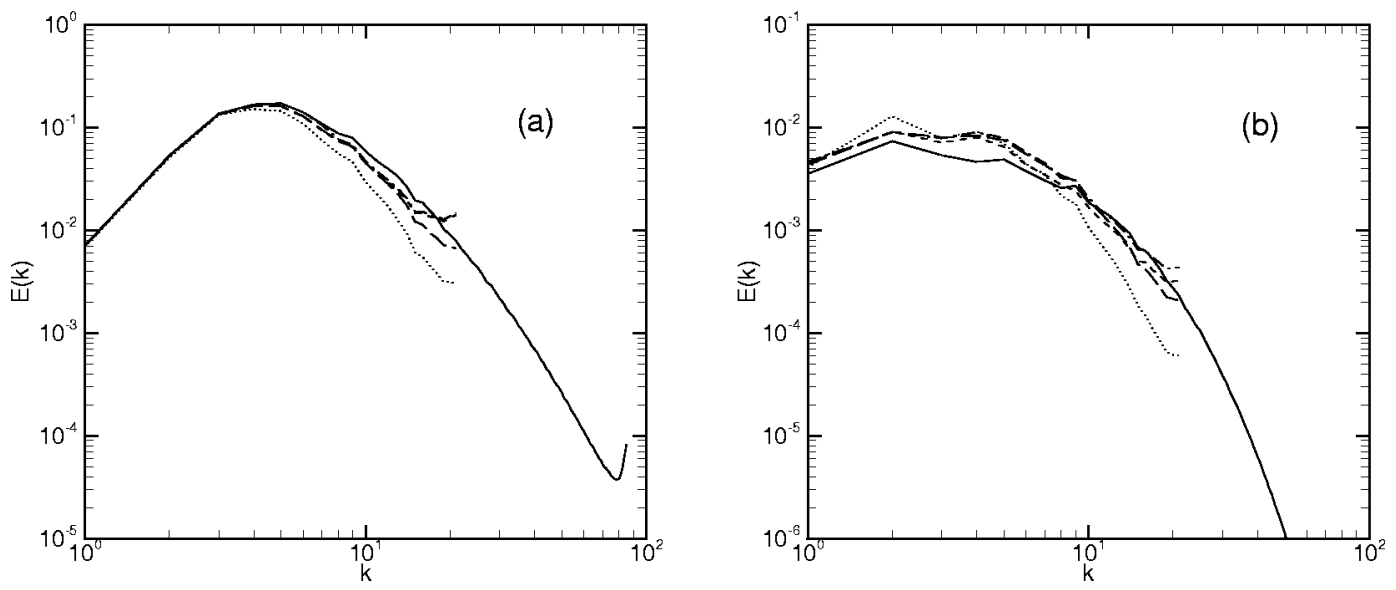

FIG. 1. Energy spectra at (a) $t=0.5$ and (b) $t=4.0 .-$, DNS; ----, dynamic Smagorinsky model; —- —, multiscale LES; $\cdots \cdots$, , Smagorinsky model; —- — spectral eddy-viscosity model.

Figure 3 plots the un-normalized time correlations of the velocity fields from the DNS and LES for wavenumbers $k$ $=5,9,13$, and 17 , spanning a range of scales from the integral scale to the lower end of the resolved scale. The starting time is $t=0.5$. A comparison clearly shows that there exist discrepancies between the LES and DNS results, and that the discrepancies become larger with increasing wavenumber. The relative performances of the models are similar to those observed in energy and energy spectra (cf. Figs. 1 and 2). The classic Smagorinsky model results are again the least accurate of all models, and the multiscale LES is the most accurate. The dynamic Smagorinsky model and spectral eddy-viscosity model yield comparable results for the first two wavenumbers, but the former is significantly more accurate at the two higher wavenumbers.

Figure 4 plots the same time correlations as in Fig. 3 but with a different starting time $t=1.5$. The discrepancies observed are qualitatively the same as in the $t=0.5$ case, except for the lowest wavenumber $k=5$ at which the correlation magnitude is overpredicted by LES, and the multiscale LES gives the largest overprediction. Overall, the SGS modeling errors are found to equally affect the time correlations in the final decay range.

In summary, it is observed in decaying isotropic turbulence that discrepancies exist between the un-normalized time correlations calculated from DNS and those from the LES. The multiscale LES approach, in conjunction with the dynamic SGS model, provides the best overall results. This is consistent with its superior prediction of the wavenumber energy spectra. Note that the multiscale LES is a methodology rather than a model, and the time correlations computed using the multiscale LES is strongly dependent on the SGS model employed. In an earlier study, ${ }^{26}$ the constantcoefficient Smagorinsky model was used in the multiscale LES, and the results were found to be less accurate compared to those obtained using LES with the dynamic SGS model.

In the following section, the computed time correlations are analyzed in the framework of Kraichnan's sweeping hypothesis, ${ }^{15}$ in order to explain the discrepancies between the LES and DNS time correlations and identify the sources of these discrepancies.

\section{ANALYSIS OF NUMERICAL RESULTS}

The analysis is based on the generalized sweeping hypothesis for decaying turbulence. In the sweeping hypothesis for stationary isotropic turbulence, the convection velocity is constant. ${ }^{15}$ However, in decaying turbulence, the convection velocity varies with time. A generalization can be made by introducing a time-dependent convection velocity, which evolves slowly relative to the time scales of velocity fluctuations.

Consider a fluctuating velocity Fourier mode $\mathbf{u}(\mathbf{k}, t)$ convected by a large-scale velocity field $\mathbf{v}(t)$. We assume that the wavenumbers $\mathbf{k}$ of the fluctuating velocity are sufficiently large. The flow scales associated with these wavenumbers are small, over which the convection velocity is spatially uniform and relatively large in magnitude. In this case, the convection effect is dominant. The governing equation for the fluctuating velocity modes is therefore

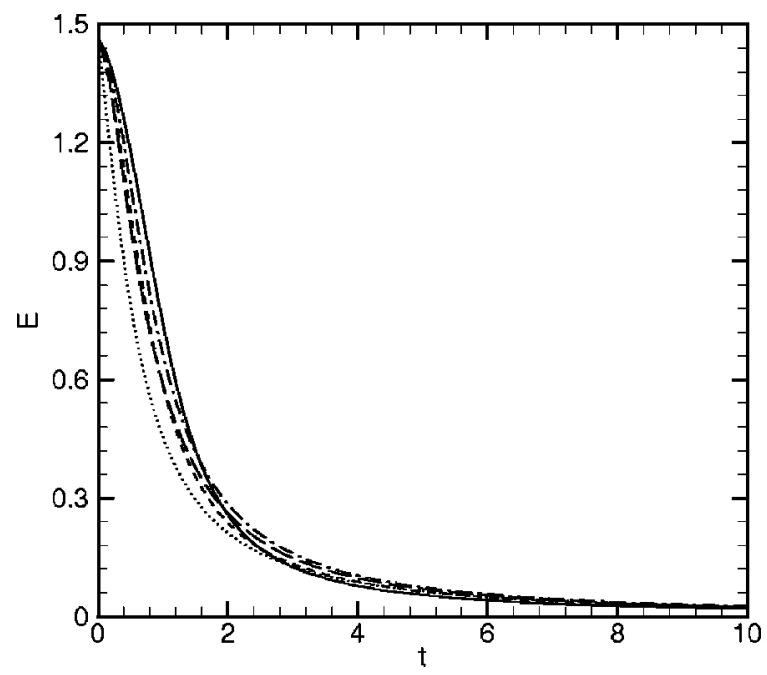

FIG. 2. Decay of total resolved energy. - DNS; ----, dynamic Smagorinsky model; —- - multiscale LES; $\cdots \cdots$, Smagorinsky model; $\_\ldots$, spectral eddy-viscosity model. 

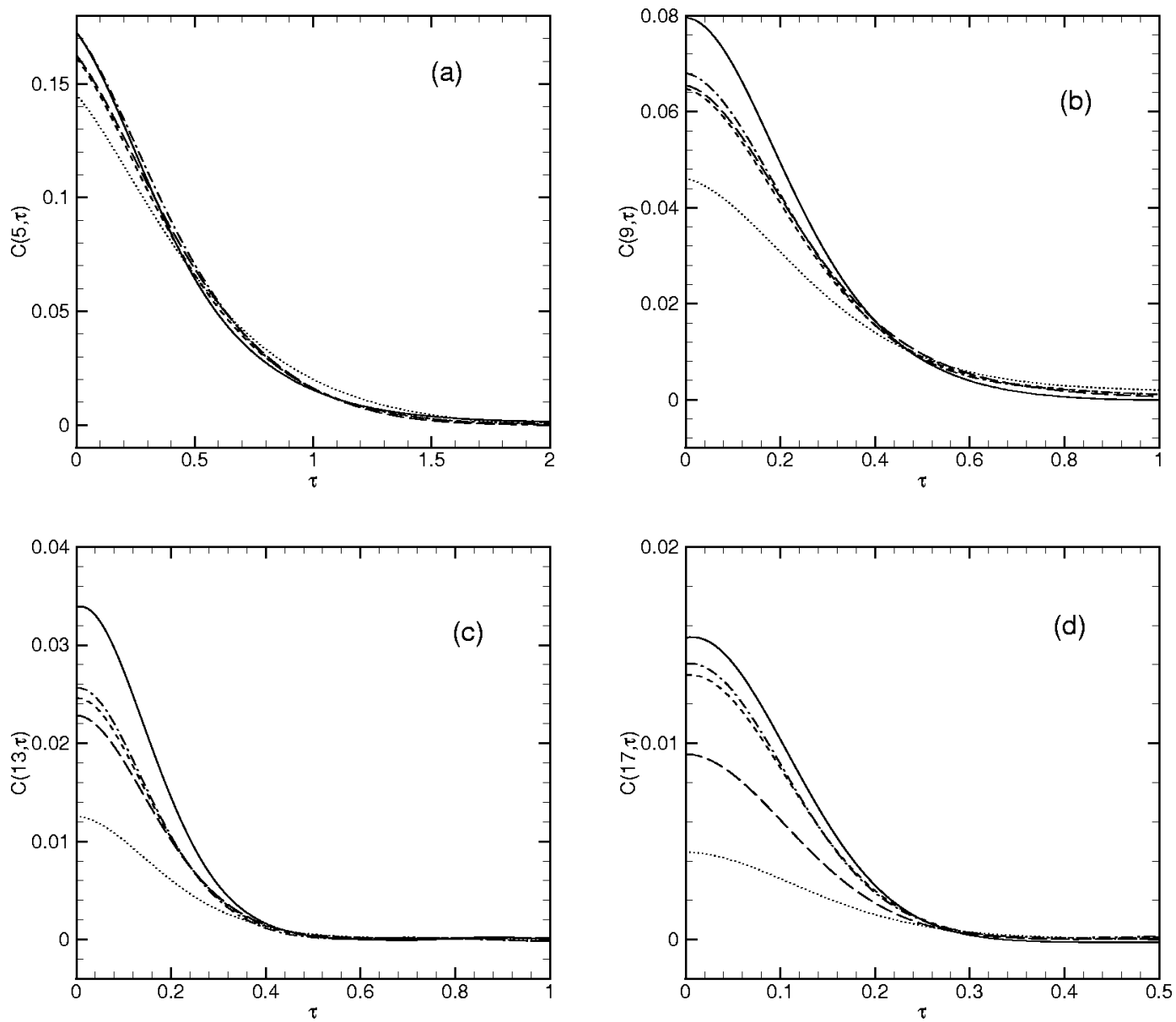

FIG. 3. Time correlation $C(k, \tau)$ vs time lag $\tau$ with starting time $t=0.5$ for (a) $k=5$, (b) $k=9$, (c) $k=13$, (d) $k=17$. —, DNS; ----, dynamic Smagorinsky model; —.-, multiscale LES; $\cdots \cdots$, Smagorinsky model; — — , spectral eddy-viscosity model.

$$
\frac{\partial \mathbf{u}(\mathbf{k}, t)}{\partial t}+i[\mathbf{k} \cdot \mathbf{v}(t)] \mathbf{u}(\mathbf{k}, t)=0
$$

which yields

$$
\mathbf{u}(\mathbf{k}, t+\tau)=\mathbf{u}(\mathbf{k}, t) \exp \left(-i \int_{t}^{t+\tau} \mathbf{k} \cdot \mathbf{v}(s) d s\right)
$$

Then, the time correlation can be expressed by

$$
\begin{aligned}
\langle\mathbf{u}(\mathbf{k}, t & +\tau) \mathbf{u}(-\mathbf{k}, t)\rangle \\
= & \langle\mathbf{u}(\mathbf{k}, t) \mathbf{u}(-\mathbf{k}, t)\rangle \\
\quad & \quad \exp \left(-\frac{1}{2} k^{2} \int_{t}^{t+\tau} \int_{t}^{t+\tau}\left\langle\mathbf{v}\left(s^{\prime}\right) \mathbf{v}\left(s^{\prime \prime}\right)\right\rangle d s^{\prime} d s^{\prime \prime}\right) .
\end{aligned}
$$

In the derivation of (6), the convection velocity $\mathbf{v}(t)$ is assumed to be Gaussian and independent of the velocity $\mathbf{u}(\mathbf{x}, t)$ at the starting time $t$. These assumptions can be justified by the near Gaussianity of the large-scale velocity and its initial independence of the small-scale velocity. By introducing a sweeping velocity

$$
V^{2}(t, \tau)=\frac{1}{\tau^{2}} \int_{t}^{t+\tau} \int_{t}^{t+\tau}\left\langle\mathbf{v}\left(s^{\prime}\right) \mathbf{v}\left(s^{\prime \prime}\right)\right\rangle d s^{\prime} d s^{\prime \prime},
$$

we obtain a general expression of time correlation similar to the one in stationary turbulence

$$
\begin{aligned}
\langle\mathbf{u}(\mathbf{k}, t+\tau) \mathbf{u}(-\mathbf{k}, t)\rangle= & \langle\mathbf{u}(\mathbf{k}, t) \mathbf{u}(-\mathbf{k}, t)\rangle \\
& \times \exp \left[-\frac{1}{2} k^{2} V^{2}(t, \tau) \tau^{2}\right] .
\end{aligned}
$$

The calculation of the sweeping velocity, (7), can be further simplified by assuming the following form of the bulk velocity correlation: ${ }^{27}$

$$
\left\langle\mathbf{v}\left(s^{\prime}\right) \mathbf{v}\left(s^{\prime \prime}\right)\right\rangle=\left\langle\mathbf{v}^{2}\left(s^{\prime}\right)\right\rangle \exp \left(-\lambda\left|s^{\prime}-s^{\prime \prime}\right|\right),
$$

where $\lambda^{-1}$ is a decorrelation time scale. Substituting (9) into (7), we find

$$
\begin{aligned}
V^{2}(t, \tau)= & \frac{1}{\tau^{2}} \int_{t}^{t+\tau}\left\langle\mathbf{v}^{2}\left(s^{\prime}\right)\right\rangle \lambda^{-1}\left(2-\exp \left[-\lambda\left(s^{\prime}-t\right)\right]\right. \\
& \left.-\exp \left[-\lambda\left(t+\tau-s^{\prime}\right)\right]\right) d s^{\prime} .
\end{aligned}
$$

In isotropic turbulence, the bulk velocity is determined by large scale motions. Hence, its decorrelation time scale $\lambda^{-1}$ is much larger than those of velocity fluctuation modes 

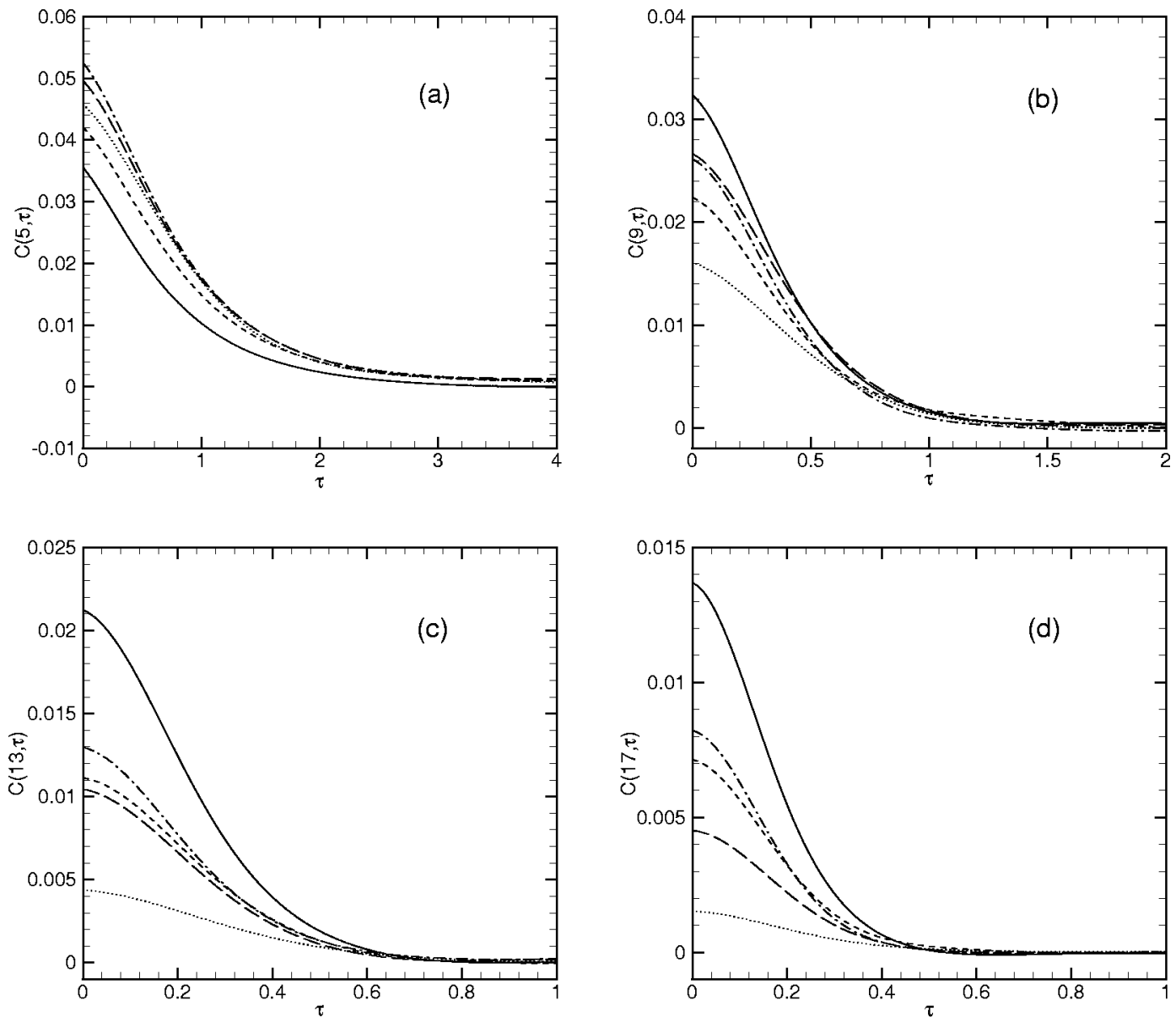

FIG. 4. Time correlation $C(k, \tau)$ vs time lag $\tau$ with starting time $t=1.5$ for (a) $k=5$, (b) $k=9$, (c) $k=13$, (d) $k=17$. —, DNS; ----, dynamic Smagorinsky model; —.-, multiscale LES; $\cdots \cdots$, Smagorinsky model; _ _ _, spectral eddy-viscosity model.

considered here. Since the time separation $\tau$ of interest is within the decorrelation time scales of the velocity fluctuations, we have $\lambda \tau \ll 1$. Using Taylor series expansion with respect to $\lambda \tau$ and ignoring the second- and higher-order terms in (10), we obtain

$$
V^{2}(t, \tau)=\frac{1}{\tau} \int_{t}^{t+\tau}\left\langle\mathbf{v}^{2}\left(s^{\prime}\right)\right\rangle d s^{\prime}
$$

Note that the bulk velocity is associated with the energycontaining motions, and its variance $\left\langle\mathbf{v}^{2}(t)\right\rangle$ is the total energy. Hence, the sweeping velocity depends on the time history of the total energy. Since the energy decay is relatively small over the decorrelation time scale, the sweeping velocity can be simply approximated by $V^{2}(t, \tau) \cong\left[\left\langle\mathbf{v}^{2}(t)\right\rangle+\left\langle\mathbf{v}^{2}(t\right.\right.$ $+\tau)\rangle] / 2$.

Figure 5 plots the normalized time correlations $R(k, \tau)$ from DNS for wavenumbers $k=5,9,13,17,30,40,50,60$, 70 , and 80 , where the correlations are normalized by the instantaneous energy spectra at the starting time $t=0.5$. The time separation is un-normalized in Fig. 5(a) and normalized by the scale-dependent similarity variable $V k$ in Fig. 5(b). The latter figure exhibits that, with the time normalization, virtually all curves collapse. The small deviation for the $k$ $=5$ curve arises because the length scale associated with this wavenumber is close to the scale of the sweeping motion, so that the sweeping hypothesis is less accurate. The results in Fig. 5(b) verifies the general validity of the sweeping hypothesis and the generalized sweeping velocity in decaying turbulence.

Equation (8) indicates that for given $k$, the normalized time correlations are solely determined by the sweeping velocities. In the present LES, the sweeping velocities are somewhat smaller than their DNS counterparts because of the reduced total energy. Therefore, the time correlations in LES decay more slowly than the ones in DNS. That is, the LES overpredicts the decorrelation time scales compared to DNS. Figure 6 plots the normalized time correlations from the DNS and LES with respect to the un-normalized time for the modes $k=5,9,13$, and 17. It confirms the overprediction of decorrelation time scales by LES, although the amount of overprediction is relatively small. Again, the multiscale LES method with dynamic SGS model is the most accurate and represents a modest improvement over the standard LES with the dynamic model. The classic Smagorinsky model is the least accurate of all the models tested. The spectral eddy viscosity model trails the dynamic model slightly.

Equation (8) also indicates that if the time separation is normalized by $V k$, the un-normalized time correlations are solely determined by the instantaneous energy spectra at the starting time. Figure 7 plots the un-normalized correlations 

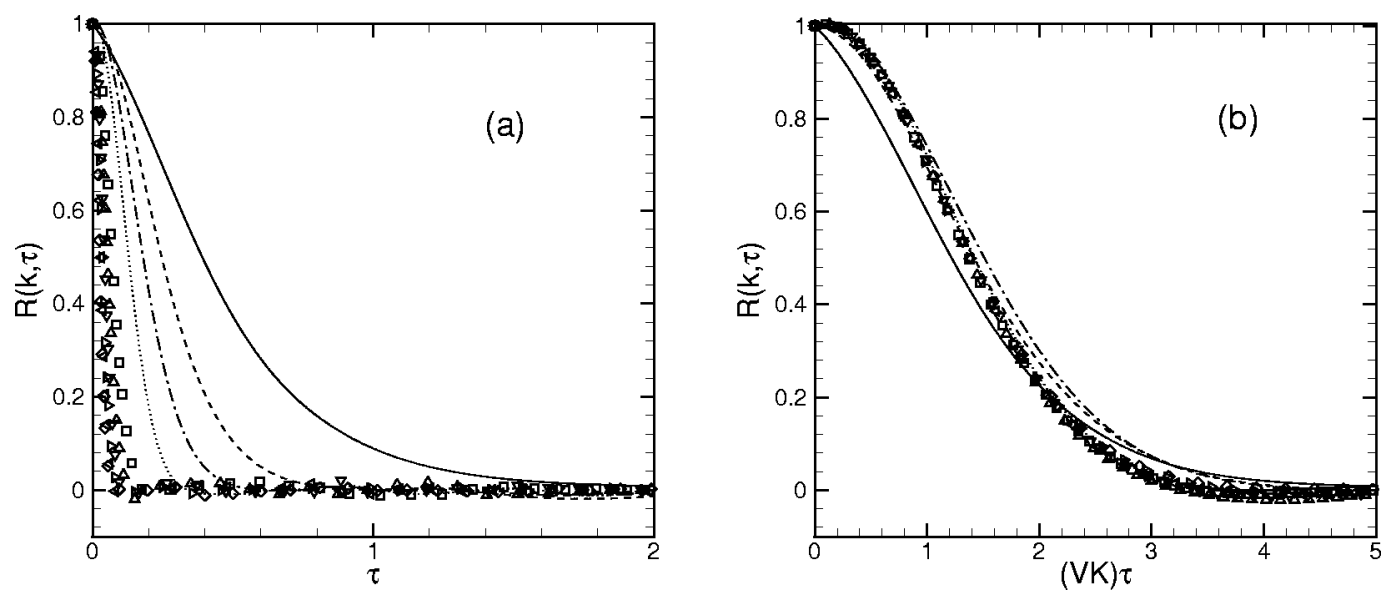

FIG. 5. Normalized time correlation $R(k, \tau)$ vs (a) un-normalized and (b) normalized time lag, with starting time $t=0.5$, for different Fourier modes computed using DNS. - $k=5 ;----, K=9 ;-.-, k=13 ; \cdots \cdots, k=17 ; \square, k=30 ; \triangle, k=40 ; \nabla, k=50 ; \triangleright, k=60 ; \triangleleft, k=70 ; \diamond, k=80$.

vs the normalized time separation. It shows that LES underestimates the magnitudes of time correlations relative to the DNS results. The underestimation becomes more significant as the wavenumber increases, which is consistent with the more severe drops of the LES energy spectra at high wavenumbers. Again, the relative performance of the SGS models
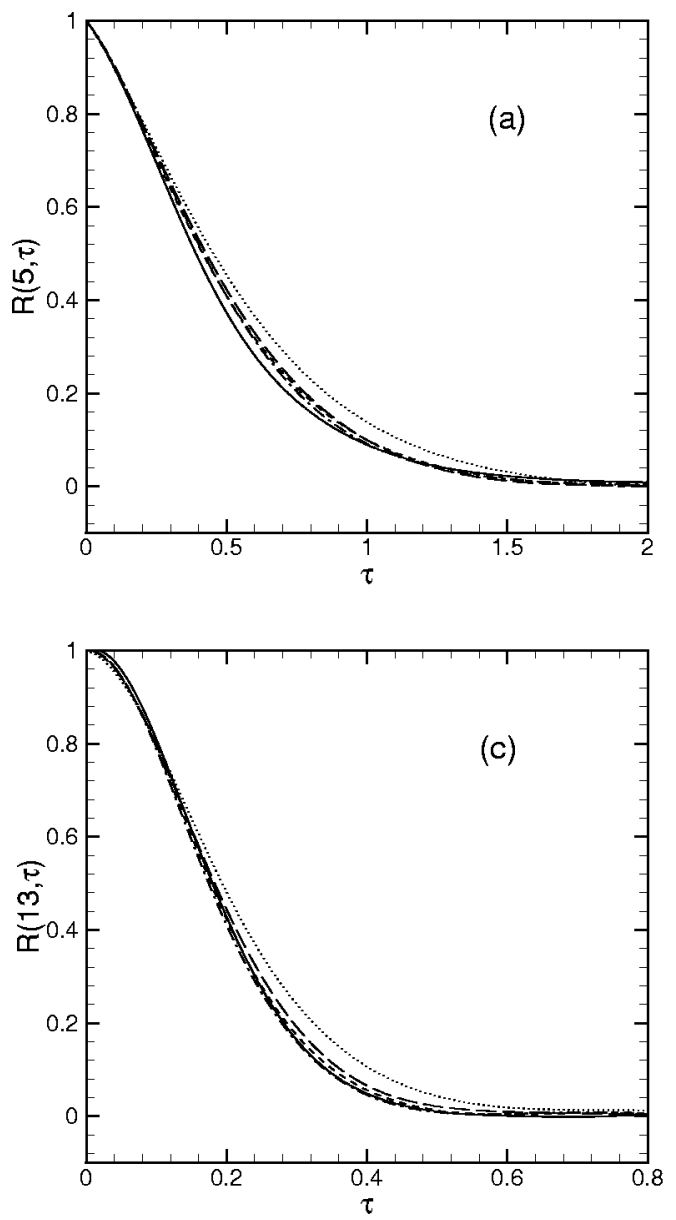

in terms of the magnitudes of time correlations is the same as before.

In conclusion, the discrepancies between the time correlations computed using DNS and LES consist of two parts: the correlation magnitude and decorrelation time scale. The errors in decorrelation time scales are induced by the sweep-
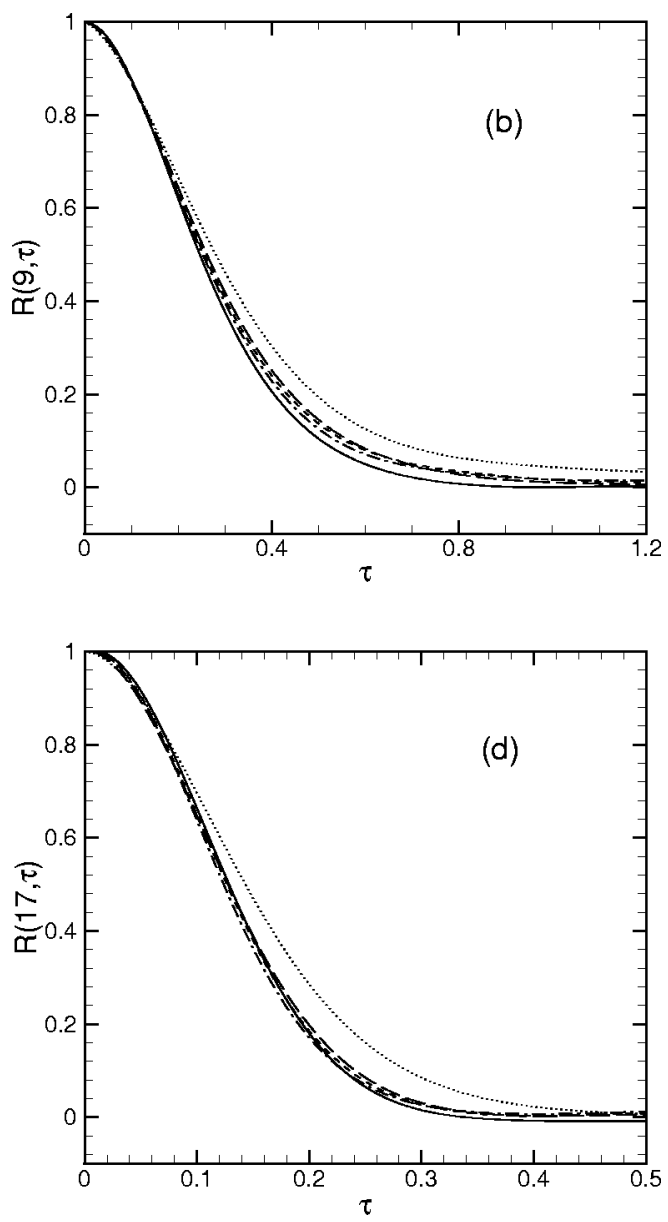

FIG. 6. Normalized time correlation $R(k, \tau)$ vs time lag $\tau$ with starting time $t=0.5$ for (a) $k=5$, (b) $k=9$, (c) $k=13$, (d) $k=17$. - , DNS; ----, dynamic Smagorinsky model; —-, multiscale LES; $\cdots \cdots$, Smagorinsky model; ———, spectral eddy-viscosity model. 

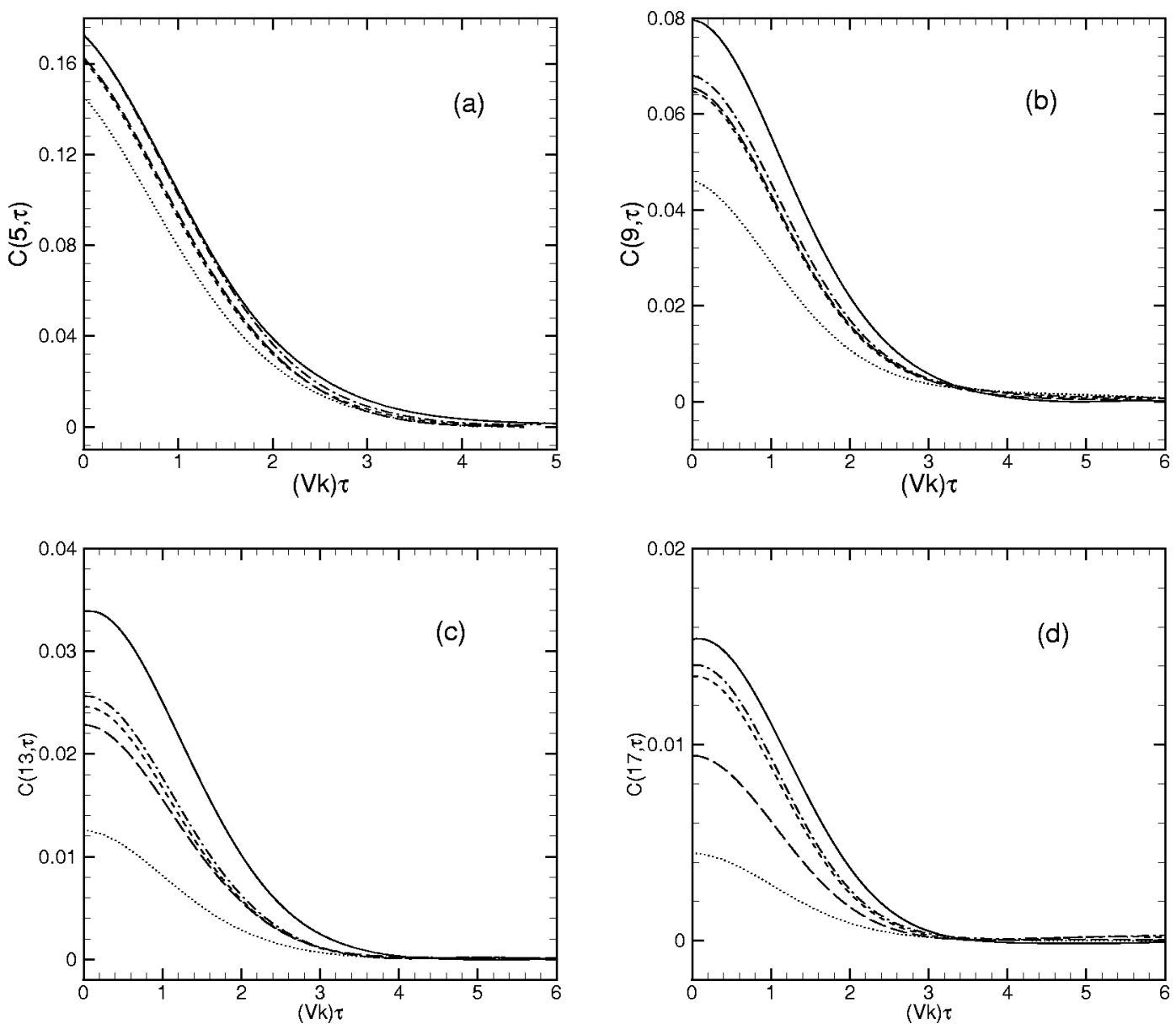

FIG. 7. Un-normalized time correlation $C(k, \tau)$ vs the normalized time lag $\tau$ with starting time $t=0.5$ for (a) $k=5$, (b) $k=9$, (c) $k=13$, (d) $k=17$. - , DNS; ----, dynamic Smagorinsky model; —. - multiscale LES; $\cdots \cdots$, Smagorinsky model; ———, spectral eddy-viscosity model.

ing velocity, and the errors in magnitudes are induced by the energy spectra. In relative terms, the errors in decorrelation time scales are less significant than those in magnitudes. However, they should not be ignored since the sound power spectra are sensitive to the decorrelation time scale (see discussions in the following section). Note that the sweeping velocity used in our analysis is the root mean square of velocity fluctuations, or the square root of the total energy. Thus, an accurate prediction of the instantaneous energy spectra is critical to the accurate computation of the time correlations. In the previous study ${ }^{10}$ in forced isotropic turbulence, a significantly larger overprediction by LES of the decorrelation time scales was observed, in contradiction with the mild overprediction estimated by the theoretical analysis presented in the same study. This is largely due to disparate total energy levels in the DNS and LES. The much smaller overprediction of decorrelation time scales by the present LES is more in line with the theoretical analysis in Ref. 10.

\section{DISCUSSION}

As an example of applications, the effect of timecorrelation errors on acoustic prediction is examined using an analytical expression of acoustic power spectra based on Lighthill's theory and the quasinormal closure assumption.
The analytical expression is only valid for stationary turbulence. However, reasonable inferences can be drawn for decaying turbulence through this analysis.

According to Lighthill's theory, ${ }^{1}$ the acoustic pressure in a far-field position $\mathbf{x}$ is given by

$$
p(\mathbf{x}, t)=\frac{1}{4 \pi c^{2}} \frac{x_{i} x_{j}}{|\mathbf{x}|^{3}} \int_{\Omega} d \mathbf{y} \frac{\partial^{2}}{\partial t^{2}} T_{i j}\left(\mathbf{y}, t-\frac{|\mathbf{x}-\mathbf{y}|}{c}\right),
$$

where $T_{i j}(\mathbf{y}, t) \approx \rho u_{i}(\mathbf{y}, t) u_{j}(\mathbf{y}, t)$ is the Lighthill stress tensor, $\Omega$ the source region, $\rho$ the mean far-field density, $c$ the speed of sound in the far-field, and $\mathbf{y}$ a position vector in the source field. The entropy and viscous stress terms have been neglected in the Lighthill stress, which is valid for low Mach number and reasonably high Reynolds number flows. Based on this equation and the quasinormal hypothesis, the acoustic power spectral density function can be written in the form ${ }^{28}$

$$
\begin{aligned}
P(\omega)= & \frac{\pi}{2} \rho \frac{\omega^{4}}{c^{5}} \frac{32 \pi}{15} \int_{0}^{+\infty} 4 \pi k^{2} \frac{E^{2}(k)}{\left(2 \pi k^{2}\right)^{2}} d k \frac{1}{2 \pi} \\
& \times \int_{-\infty}^{+\infty} R^{2}(k, \tau) \exp (-i \omega \tau) d \tau .
\end{aligned}
$$

In the following discussion, the normalized time correlation $R(k, \tau)$ is assumed to be of the exponential form 

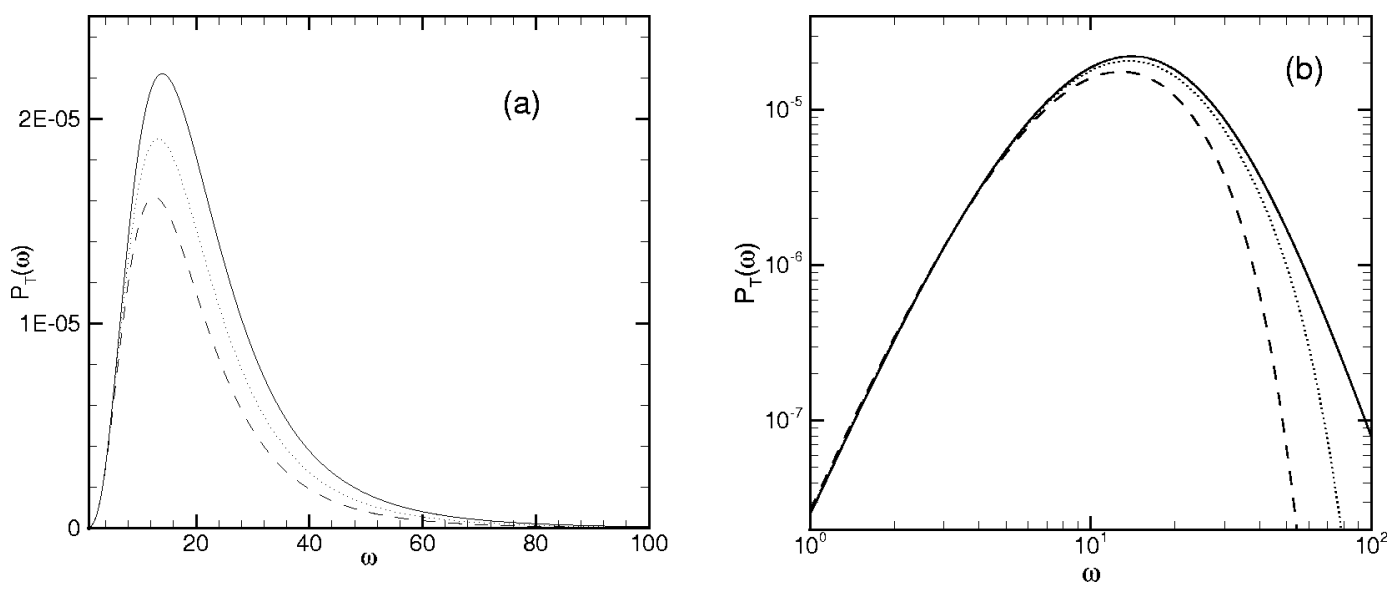

FIG. 8. Effects of (a) sweeping velocity $(-, V=1.0 ; \cdots \cdots, V=0.95 ;-\ldots-, V=0.90)$ and (b) energy spectrum truncation $\left(-\right.$, full spectra; $\cdots \cdots, k_{c}=25$; $\ldots \ldots, k_{c}=13$ ) on predicted sound power spectra. The corresponding sweeping velocities for the three cases in (b) are $V=1,0.978$, and 0.933 , respectively.

$$
R(k, \tau)=\exp \left(-\frac{1}{2} k^{2} V^{2} \tau^{2}\right),
$$

and the energy spectrum $E(k)$ is represented by the von Kármán spectrum

$$
E(k)=C \epsilon^{2 / 3} k_{0}^{-5 / 3}\left(k / k_{0}\right)^{4}\left[1+\left(k / k_{0}\right)^{2}\right]^{-17 / 6} k^{-2},
$$

where $k_{0}=5$ defines the peak of energy spectrum. The exponential form and the von Kármán spectrum are the appropriate approximations to the time correlations and the energy spectra, respectively, in our numerical simulations.

With the substitution of Eq. (14) into Eq. (13), the nondimensionalized sound power spectra are given by

$$
P_{T}(\omega)=\frac{2 \sqrt{\pi}}{15} \rho M^{5} \frac{\omega^{4}}{V} \int_{0}^{+\infty} k^{-3} E^{2}(k) \exp \left(-\frac{\omega^{2}}{4(V k)^{2}}\right) d k,
$$

where $M=V_{0} / c$ is the Mach number and $V_{0}=\omega_{0} / k_{0} . k_{0}$ is the inverse integral length scale and $\omega_{0}$ the inverse integral time scale.

The influences of decorrelation time scales on acoustic power spectra can be seen in Fig. 8(a), where the sound power spectra are evaluated according to (16) with the sweeping velocities $V$ equal to $1.0,0.95$, and 0.9 . The small variations, up to $10 \%$, of the sweeping velocities cause significant reductions of the sound power spectra at higher frequencies. This illustrates the sensitivity of the acoustic power spectra to the sweeping velocities.

The sweeping-velocity induced errors can be compounded by the truncation of the energy spectra at high wavenumbers, corresponding to unresolved scales in LES. To test this effect, the energy spectrum is truncated $[E(k)$ set to zero] for either $k>25$ or $k>13$. These truncations correspond to grid-size ratios of 1:4 and 1:8, respectively, between LES and DNS. The sweeping velocities, computed based on the respective truncated energy spectra, are 0.978 and 0.933 compared to 1 for DNS. Figure 8(b) plots the acoustic power spectra calculated using the full and truncated energy spectra. It shows that in the truncated cases, the acoustic spectra drop considerably at moderate to high frequencies, and the spectral peaks are shifted towards left to lower frequencies.
It should be noted that the above assessment is based on a model energy spectrum, and therefore should be viewed in a qualitative sense. At low wavenumbers, the correlation function expression (14) based on sweeping hypothesis may not be appropriate. Furthermore, it is generally considered that noise generation by turbulent flows is predominantly through the generation and nonlinear interaction of turbulent eddies, which may not be adequately analyzed using the sweeping hypothesis. A more systematic evaluation of the acoustic power spectra will be pursued in the future in order to quantify the SGS modeling effects on aeroacoustic predictions.

\section{CONCLUSIONS}

Numerical comparisons in decaying isotropic turbulence suggest that there exist discrepancies in time correlations evaluated by DNS and LES using eddy-viscosity-type SGS models. This is qualitatively consistent with the previous observations in forced isotropic turbulence. Comparisons among different SGS models in the LES also indicate that the model choice affects the time correlations. The dynamic Smagorinsky model provides significantly more accurate predictions than the classic Smagorinsky model and slightly more accurate predictions than the spectral eddy-viscosity model. The multiscale LES using the dynamic Smagorinsky model on the small scale equations is shown to be the most accurate approach.

The generalized sweeping hypothesis implies that time correlations in decaying isotropic turbulence are mainly determined by the energy spectra and sweeping velocities. The analysis based on the sweeping hypothesis explains the discrepancies in our numerical simulations: the LES underpredicts the magnitudes of time correlations because the energy spectrum levels are lower than the DNS values, and slightly overpredicts the decorrelation time scales because the sweeping velocities are smaller than the DNS values. Since the sweeping velocity is determined by the energy spectra, one concludes that an accurate prediction of the time history of the energy spectra guarantees the accuracy of time correlations. Note that the generalized sweeping hypothesis itself 
does not explain the relative performance of the various SGS models for space-time correlations. Rather, it explains their accuracy in terms of their ability to predict the instantaneous energy spectra, which is a simpler criterion.

As an example, the effect of time-correlation errors on radiared sound power spectra is estimated based on Lighthill's theory and the quasinormal closure assumption. It is shown that smaller sweeping velocities and energy spectrum truncation can cause significant errors in the sound power spectra, which exhibit a sizable drop at moderate to high frequencies accompanied by a shift of the peaks to lower frequencies. Based on this analysis, two possible ways to improve acoustic predictions can be considered. The first is to construct better SGS models to improve the LES accuracy for time correlations. The second is to remedy the temporal statistics of the Lighthill stress tensor in order to "recover" the contribution from the unresolved scales in LES to time correlations.

\section{ACKNOWLEDGMENTS}

We wish to thank Professor P. Moin, Dr. A. Wray, Dr. D. Carati, and Dr. R. Rubinstein for helpful discussions. G.-W.H.'s work was partially supported by the Special Funds for Major Basic Research, Project No. G2000077305, People's Republic of China, and National Natural Science Foundation of China under Project No. 10325211. M.W. acknowledges support from ONR under Grant No. N00014-01-1-0423.

${ }^{1}$ M. J. Lighthill, "On sound generated aerodynamically: I. General theory," Proc. R. Soc. London, Ser. A 211, 564 (1952).

${ }^{2}$ I. Proudman, "The generation of sound by isotropic turbulence," Proc. R. Soc. London, Ser. A 214, 119 (1952).

${ }^{3}$ W. K. Blake, Mechanics of Flow-Induced Sound and Vibration (Academic, London, 1986), Vols. I and II.

${ }^{4}$ W. S. Saric, H. L. Reed, and E. J. Kerschen, "Boundary-layer receptivity to freestream disturbance," Annu. Rev. Fluid Mech. 34, 291 (2002).

${ }^{5}$ J. Lumley and P. Blossey, "Control of turbulence," Annu. Rev. Fluid Mech. 30, 311 (1998).

${ }^{6}$ G. Falkovish, K. Gawedzki, and M. Vergassola, "Particles and fields in fluid turbulence," Rev. Mod. Phys. 73, 913 (2001).
${ }^{7}$ C. E. Leith and R. H. Kraichnan, "Predictability of turbulent flows," J. Atmos. Sci. 29, 1041 (1972).

${ }^{8}$ C. Meneveau and J. Katz, "Scale-invariance and turbulence models for large-eddy simulation," Annu. Rev. Fluid Mech. 32, 1 (2000).

${ }^{9}$ D. G. Crighton, "Basic principles of aerodynamic noise generation," Prog. Aerosp. Sci. 16, 31 (1975).

${ }^{10}$ G.-W. He, R. Rubinstein, and L.-P. Wang, "Effects of subgrid scale modeling on time correlations in large-eddy simulation," Phys. Fluids 14, 2186 (2002).

${ }^{11}$ J.-P. Chollet and M. Lesieur, "Parametrization of small scales of threedimensional isotropic turbulence utilizing spectral closure," J. Atmos. Sci. 38, 2747 (1981).

${ }^{12}$ J. Smagorinsky, "General circulation experiments with the primitive equations: I. The basic experiment," Mon. Weather Rev. 91, 99 (1963).

${ }^{13}$ M. Germano, U. Piomelli, P. Moin, and W. H. Cabot, "A dynamic subgridscale eddy viscosity model," Phys. Fluids A 3, 1760 (1991).

${ }^{14}$ T. J. R. Hughes, L. Mazzei, and A. S. Oberai, "The multiscale formulation of large-eddy simulation: Decay of homogeneous isotropic turbulence," Phys. Fluids 13, 505 (2001).

${ }^{15}$ R. H. Kraichnan, "Kolmogorov's hypotheses and Eulerian turbulence theory," Phys. Fluids 7, 1723 (1964).

${ }^{16} \mathrm{Y}$. Kaneda and T. Gotoh, "Lagrangian velocity autocorrelation in isotropic turbulence,” Phys. Fluids A 3, 1924 (1991).

${ }^{17}$ Y. Kaneda, "Lagrangian and Eulerian time correlations in turbulence," Phys. Fluids A 5, 2835 (1993).

${ }^{18}$ R. Rubinstein and Y. Zhou, "The frequency spectrum of sound radiated by isotropic turbulence," Phys. Lett. A 267, 379 (2000).

${ }^{19} \mathrm{M}$. Wang and P. Moin, "Computation of trailing-edge flow and noise using large-eddy simulation,” AIAA J. 38, 2201 (2000).

${ }^{20}$ U. Piomelli, C. L. Streett, and S. Sarkar, "On the computation of sound by large-eddy simulation,” J. Eng. Math. 32, 217 (1997).

${ }^{21}$ A. Witkowska, D. Juvé, and J. M. Brasseur, "Numerical study of noise from isotropic turbulence," J. Comput. Acoust. 5, 317 (1997).

${ }^{22}$ C. Seror, P. Sagaut, C. Bailly, and D. Juvé, "Subgrid-scale contribution to noise production in decaying turbulence," AIAA J. 38, 1795 (2000).

${ }^{23}$ C. Seror, P. Sagaut, C. Bailly, and D. Juvé, "On the radiated noise computed by large-eddy simulation," Phys. Fluids 13, 476 (2001).

${ }^{24}$ D. G. Crighton, in Computational Aeroacoustics, ICASE/NASA LaRC Series, edited by J. C. Hardin and M. Y. Hussaini (Springer, New York, 1993), pp. 50-68.

${ }^{25}$ M. Wang, S. K. Lele, and P. Moin, "Computation of quadrapole noise using acoustic analogy," AIAA J. 24, 2247 (1996).

${ }^{26}$ G.-W. He, M. Wang, and S. K. Lele, "Evaluation of subgrid scale models in terms of time correlations," in Proceedings of the Summer Program (Center for Turbulence Research, NASA Ames/Stanford University, Stanford, CA, 2002), pp. 73-78.

${ }^{27}$ D. C. Leslie, Developments in the Theory of Turbulence (Clarendon, Oxford, 1973).

${ }^{28}$ R. Rubinstein and Y. Zhou, "Characterization of sound radiation by unresolved scales of motion in computational aeroacoustics," Eur. J. Mech. B/Fluids 21, 105 (2002). 